\title{
Los contratos de consumo en Colombia
}

Consumer contracts in Colombia

Os contratos de consumo na Colômbia

Hermes Fernando Rico Charry ${ }^{1}$

\begin{abstract}
Recibido: Febrero 16 de 2019
Aprobado: Abril 27 de 2019

Publicado: 11 de noviembre de 2019

Cómo citar este artículo:

Hermes Fernando Rico Charry, Los contratos de consumo en Colombia. DIXI 29,

Enero 2019, 1-17.

DOI: https://doi.org/10.16925/2357-5891.2019.01.04
\end{abstract}

Artículo de investigación. https://doi.org/10.16925/2357-5891.2019.01.04

1 Candidato a posdoctor en Ciencias de la Educación, magíster en Dirección de Marketing, especialista en Derecho Administrativo, especialista en Legislación Energética y Telecomunicación, especialista en Gerencia Financiera, especialista en Gestión de Empresa, especialista en Gerencia de Proyectos, abogado.

ORCID: https://orcid.org/0000-0001-6672-048X

Correo electrónico: hricocha@uniminuto.edu.co 


\section{Resumen}

Objetivo: el objetivo general del presente artículo fue realizar un análisis sobre el contrato en general y el contrato de consumo en particular, a través de una investigación propositiva que se basó en una metodología dogmática y en un análisis doctrinal, jurisprudencial y legislativo.

Metodología: a partir del análisis propuesto, se expondrá el estudio de diferentes conceptos y posiciones sobre los contratos y sus elementos de formación: la oferta, la aceptación y el perfeccionamiento del contrato, para después abordar el contrato de consumo, sus características como contrato asimétrico y su relación con el contrato de adhesión, finalizando con una conceptualizacion del contrato de consumo como un contrato normado.

Conclusiones: el perfeccionamiento del contrato requiere cumplir con las condiciones de la promesa y con las formalidades que ha estipulado la ley para cada caso de contrato. Los contratos de consumo son asimétricos, porque el poder económico y la información son detentados por los productores o comerciantes frente a los consumidores. A su vez, el contrato de consumo es un contrato normado, con un nivel de protección especial debido a que no existe igualdad alguna entre los contratantes, pero esta protección es insuficiente, dada la dinámica de las formas de ofrecer y comprar bienes de consumo, que evoluciona hasta el punto de que la legislación al respecto, generalmente, está atrasada para generar el desequilibrio entre productores y/o comercializadores y consumidores.

Palabras clave: aceptación, contrato, contrato asimétrico, contrato de adhesión, contrato de consumo, contrato normado, oferta, perfeccionamiento del contrato.

\section{Abstract}

Objective: The general objective of this article is to carry out an analysis of contracts in general, and consumer contracts in particular, through a proactive investigation based on a dogmatic methodology and a doctrinal, jurisprudential and legislative analysis.

Methodology: Based on the proposed analysis, the study of different concepts and positions on contracts and their background elements will be presented: the offer, acceptance and improvement of the contract; the consumer contract will then be addressed, its characteristics as an asymmetric contract and its relationship with the adhesion contract, ending with a conceptualization of the consumer contract as a standard contract.

Conclusions: The completion of the contract requires compliance with the conditions of the undertaking and with the formalities established by law for each contract case. Consumer contracts are asymmetric, because where consumers are concerned, economic power and information are held by the producers or merchants. In turn, the consumer contract is a regulated contract, with a special level of protection because there is no equality between the contracting parties. This protection is insufficient, given the dynamics of the forms of offering and buying consumer goods, which evolves to the point that the legislation in this regard, generally, is behind schedule, generating an imbalance between producers or marketers and consumers.

Keywords: acceptance, contract, asymmetric contract, adhesion contract, consumer contract, standard contract, offer, completion of the contract.

\section{Resumo}

Objetivo: o objetivo geral do presente artigo foi realizar uma análise sobre o contrato em geral e sobre o contrato de consumo em particular por meio de uma pesquisa propositiva com base em uma metodologia dogmática e em uma análise doutrinal, jurisprudencial e legislativa. 
Metodologia: a partir da análise proposta, será exposto o estudo de diferentes conceitos e posições sobre os contratos e seus elementos de formação: a oferta, a aceitação e o aperfeiçoamento do contrato, para abordar posteriormente o contrato de consumo, suas características como contrato assimétrico e sua relação com o contrato de adesão, e finaliza-se com uma conceitualização do contrato de consumo como um contrato atípico.

Conclusões: o aperfeiçoamento do contrato requer o cumprimento das condições da promessa e das formalidades estipuladas por lei para cada tipo de contrato. Os contratos de consumo são assimétricos porque o poder econômico e a informação são apropriados pelos produtores ou comerciantes em detrimento dos consumidores. Por sua vez, o contrato de consumo é um contrato atípico com um nível de proteção especial devido ao fato de que não existe igualdade nenhuma entre os contratantes, mas essa proteção é insuficiente dada a dinâmica das formas de oferecer e comprar bens de consumo, que evolui até o ponto em que a respectiva legislação, geralmente, está atrasada para gerar o desequilíbrio entre produtores e/ou comercializadores e consumidores.

Palavras-chave: aceitação, contrato, contrato assimétrico, contrato de adesão, contrato de consumo, contrato atípico, oferta, aperfeiçoamento do contrato.

\section{INTRODUCCIÓN}

Los contratos de consumo en Colombia, como sucede en todos los países, generan unos efectos jurídicos a los que las personas deben someterse, y que se contextualizan en un marco de bilateralidad entre productores y/o consumidores y los compradores o consumidores.

Es importante tener en cuenta que en Colombia muchos consumidores desconocen la naturaleza de los contratos; de hecho, desconocen que toda compra de un bien o un servicio es un contrato y esto hace que muchos consumidores acepten recibir bienes y servicios, consintiendo su compra, sin conocer que no tienen posibilidad de devolverlos o que teniendo dicha oportunidad, no la surtan o no lo hagan en los términos fijados por la ley.

Por ello, el objetivo general del presente artículo fue realizar un análisis sobre el contrato en general y el contrato de consumo en particular, a través de una investigación propositiva que se basó en una metodología dogmática y en un análisis doctrinal, jurisprudencial y legislativo.

A partir del análisis propuesto, se expondrá el estudio de diferentes conceptos y posiciones sobre los contratos y sus elementos de formación: la oferta, la aceptación y el perfeccionamiento del contrato, para después abordar el contrato de consumo, sus características como contrato asimétrico y su relación con el contrato de adhesión, finalizando con una conceptualizacion del contrato de consumo como un contrato normado. 


\section{MeTOdología deL ESTUDIO}

La investigación fue propositiva porque pretendía, a partir de la aplicación de la metodología dogmática, profundizar en los contratos de consumo como generadores de obligaciones según las normas establecidas en el Código Civil, en el Código de Comercio y en las sentencias de la Corte.

El estudio partió de la información bibliográfica y en red: textos, artículos y documentos que ofrecían información sobre contratos en general y contratos de consumo en particular. Para el desarrollo del proceso investigativo, fue necesario fundamentar la exposición de conceptos y doctrinas, por lo que se procedió a recopilar la información, clasificarla y analizarla para proceder a la redacción del artículo final.

\section{FORMACIÓN DEL CONTRATO}

Para Hobbes', el contrato surge de una transferencia y en este sentido expresa: "La mutua transferencia de derechos es lo que los hombres llaman contrato". Al respecto, Puig ${ }^{2}$ considera que el contrato es toda convención o acuerdo de voluntades por el que se crean, modifican o extinguen relaciones jurídicas de contenido patrimonial y que se hallan al alcance de la autonomía de la voluntad. Con criterio más estricto, la palabra contrato hace referencia al acuerdo de voluntades de dos o más partes por el que se crean, modifican o extinguen relaciones pertenecientes al derecho de obligaciones.

Diez Picazo ${ }^{3}$ expresa que mediante el acuerdo de voluntades de dos o más, las partes crean obligaciones y derechos; y en el mismo sentido, Albadalejo ${ }^{4}$ considera que la esencia del contrato radica en el acuerdo de voluntades.

Para Scognamiglio 5 , el contrato implica obligatoriamente que su contenido sea patrimonial. De la Puente y Lavalle ${ }^{6}$ consagra que existen diferentes formas de contratos y los clasifican en cuatro grandes grupos: i) contrato como el acuerdo destinado solamente a crear obligaciones; ii) contrato como el acuerdo cuya finalidad es crear, modificar o extinguir un vínculo jurídico; iii) contrato como el acuerdo encaminado a

1 Cf. Thomas Hobbes. LEVIATÁN O LA MATERIA, FORMA Y PODER DE UNA REPÚBLICA ECLESIÁSTICA Y CIVIL. Fondo de Cultura Económica (2001).

2 Cf. José Puig Brutau. FUndamentos de DERECHo CIVIL. Marcial Pons. (1978).

3 Cf. Luis Diez Picazo. teoría del contrato (vol. 1). Civitas. (1993).

4 Cf. Manuel Albaladejo García. DERECHO CIVIL, EL NEGOCIO JURídICo. Bosch. (1994).

5 Cf. Renato Scognamiglio. TEORÍA GENERAL DEL CONTRATO. Universidad Externado de Colombia. (1996).

6 Cf. Manuel De la Puente y Lavalle. EStudios SobRE EL CONTRATO PRIVADo. Lima: T.I. (1983). 
crear, modificar o extinguir relaciones jurídicas patrimoniales; y iv) contrato como el acto o negocio jurídico y contrato como el acuerdo de dos o más partes sobre una declaración conjunta de voluntad común destinada a constituir, regular o extinguir una relación jurídica patrimonial.

Larroumet ${ }^{7}$ profundiza sobre dicho concepto y expresa que es "una relación entre partes en el proceso de programación de intercambios en el futuro", por lo que el contrato puede ir más allá de transacciones o actos del presente, pues puede decidir y consensuar sobre hechos del futuro que pueden ser objeto de intercambios. Dado que en Colombia la formación del contrato está precedida por una oferta y una aceptación, es importante profundizar sobre estos dos aspectos.

\section{Oferta}

La oferta es un acto jurídico unilateral dirigido al perfeccionamiento de un contrato. La oferta contiene los elementos esenciales del negocio jurídico que se quiere celebrar; así, quien tiene la iniciativa de contratar presenta la oferta bien porque esta es su intención o porque recibe una invitación para presentar oferta por parte de su destinatario ${ }^{8}$.

En este sentido, la oferta como invitación a contratar puede estar dirigida a una persona determinada o indeterminada, como es el caso de la policitación, en la cual debe identificarse el producto y el precio, y si la oferta está dirigida a consumidores, es obligatorio señalar su vigencia.

\section{Declaración de voluntad}

La voluntad puede ser expresa o tácita. En el primer caso, la declaración puede darse por cualquier medio de comunicación idóneo para tal fin: escrito, verbal, electrónico; en el segundo caso, se suscita por actos inequívocos que no admitan duda sobre la intención de celebrar un contrato, como es el caso de las ofertas expuestas al público en general.

Con respecto a las modalidades de la oferta, es decir, verbal o escrita, es preciso señalar que si es verbal, puede hacerse incluso telefónicamente.

7 Cf. Christian Larroumet. TeOría General del Contrato (Vol. I). Temis. (1999).

8 Cf. Manuel González Pernett. Contratos y obligaciones: LA OFERTA o PROPUESTA A CONTRATAR. (2010). Disponible en: http://migonzalezp.blogspot.com/2009/08/la-oferta.html 


\section{Encaminada a la celebración de un negocio jurídico.}

La oferta debe dirigirse a vincularse contractualmente; es decir, el oferente debe estar dispuesto a celebrarlo ante la simple aceptación por el destinatario. En este sentido, es importante tener en cuenta que no es oferta cuando se inserte una cláusula como "sin compromiso" u otra equivalente como "aplicación de condiciones y restricciones" o "sujeta a aprobación o confirmación del oferente".

Completa. La oferta debe contener todos los elementos esenciales para la celebración del contrato que se espera celebrar. Debe ser de tal manera que en los negocios consensuales, con la simple aceptación quede perfeccionado el contrato. En los que requieran alguna solemnidad, como la escritura pública, se requerirá que se agote esta para perfeccionar el contrato, pero en este caso la oferta no tiene que ser solemne porque el código la permite verbal o escrita.

\section{Comunicada al destinatario.}

Se requiere que se comunique a quien va dirigida por cualquier medio idóneo, se trate de persona determinada o indeterminada ${ }^{9}$. La oferta es autónoma, lo que quiere decir que se mantiene aunque sobrevenga la muerte o la incapacidad legal del oferente ${ }^{10}$.

Esta declaración de voluntad emitida por una persona y dirigida a otra u otras debe contener todos los elementos necesarios para la existencia del contrato pretendido, de tal suerte que acaecida la aceptación, se perfeccione el contrato y así las partes queden vinculadas. Si se presentan reservas implícitas o explícitas, como salvo confirmación o salvo aprobación, no nos encontramos frente a una oferta propiamente dicha, pues compartiendo la postura de Ruggiero, "la oferta es tan solo declaración de voluntad dirigida por una parte a otra para provocar la adhesión del destinatario a la propuesta"11.

Es importante aclarar que la oferta será eficaz en la medida en que: si es oferta verbal, sea conocida por su destinatario; si es oferta escrita, sea recibida por el receptor o se presuma que este la recibió; y si es una exhibición pública, porque contiene la información correspondiente al negocio a celebrar, esta eficacia per se genera como efecto la obligación para el oferente de respetar la oferta, razón por la cual no podrá retirarse ni modificarse, pues la oferta es irrevocable.

\section{Id.}

10 Id.

11 Cf. Roberto Ruggiero. INSTITUCIONES DE DERECHO CIVIL (TOMO II). Civitas. (1993). 


\section{La aceptación}

"La aceptación es un acto jurídico unilateral por medio del cual el destinatario acepta incondicionalmente la oferta"12. Así, en palabras de Farnsworth³ ${ }^{13}$, "la aceptación es el tramo final del periodo de formación del contrato. Esto implica que la aceptación es la culminación de un acto que se inicia con la oferta y que como parte de formación del contrato está articulada y condicionada a dicha oferta".

Para Kaune Arteaga ${ }^{14}$, la aceptación es la conformidad del destinatario frente a la consumación del negocio jurídico propuesto por el oferente, por lo que dicho acto implica consentimiento y en él se concreta el acuerdo de voluntades que requiere la formación de un contrato. Esta posición se complementa con lo expuesto por Barros ${ }^{15}$ de que "la aceptación es la adhesión de la voluntad de aquel a quien se hace una proposición u oferta". No obstante, esta adhesión solo tiene fuerza jurídica en los términos y negocios específicos de la oferta.

En este mismo sentido, tal y como ha afirmado la doctrina, es necesario que la aceptación se manifieste mientras la oferta se encuentre vigente, es decir, antes de que el oferente la revoque o dentro del plazo fijado por el oferente para su manifestación. Según Barros ${ }^{16}$, debe aceptarse antes de suscitarse supuestos, tales como la pérdida de la capacidad del oferente u otras circunstancias que determinen la caducidad de la oferta.

Incluso en la actualidad, muchas veces no media la manifestación de la voluntad como una expresión del querer del contratante, sino que se da como consecuencia de una necesidad que se requiere satisfacer y por las condiciones del mercado el aceptante solo se adhiere al contrato, situación en la que pareciera pasar a un segundo plano la voluntad.

En consonancia con lo anterior, la aceptación debe ser pura y simple. Esto implica que no requiere formalidades extremas, basta el simple asentimiento. Por el contrario, debe ser tan simple que no pueda confundirse con una aceptación condicional.

El Código de Comercio ${ }^{17}$ también consagra como características de la aceptación el hecho de que sea oportuna, porque si es extemporánea, esto es, por fuera de los términos indicados en ella o en su defecto por la ley, se tendría como una

12 Véase. M. González Pernett, supra, nota 8.

13 Cf. Allan Farnsworth. contratos (томо I). Aspen Law \& Business Publisher. (1998).

14 Cf. Walter Kaune Arteaga. CURSO DE DERECHO CIVIL DE CONTRATOS. Zegada. (1981).

15 Cf. Alfredo Barros Errazuriz. CURSO DE DERECHO civil (VOL. III). Nascimento. (1932).

16 Id.

17 Decreto 410 de 1971 [Presidencia de la República de Colombia]. Por el cual se expide el Código de Comercio. Junio 16 de 1971. 
contraoferta (artículo 855 c.co.). Igualmente: "La oferta escrita deberá ser aceptada dentro de los seis (6) días siguientes a la fecha que tenga la propuesta; si el destinatario vive en otra ciudad, se sumará el término de la distancia" (art. 851 c.co.). Por su parte, "la [aceptación] verbal deberá aceptarse en al acto de oírse. Cabe aclarar que la comunicación telefónica se asimila a la verbal" (art. 850 c.co.).

Finalmente, de acuerdo con González Pernett18: la aceptación debe ser expresa o tácita. "Es expresa cuando se realiza por cualquier medio idóneo encaminado a hacerla conocer al oferente. Será tácita cuando se realicen actos inequívocos de ejecución del contrato propuesto".

\section{Perfeccionamiento del contrato}

La legislación civil colombiana señala que la manifestación de voluntad es la base de la formación del contrato, razón por la cual no es aceptable acudir a la teoría objetiva, que está orientada a que por el comportamiento de las partes se pueda considerar perfeccionado el contrato. Sin embargo, esta explicación es suficiente tratándose de contratos entre iguales, pero desconoce las condiciones actuales del mercado, en donde una parte - mediando más necesidad que voluntad-consentimiento- se adhiere al contrato o se ve obligada a su cumplimiento.

No obstante, los contratos solemnes requieren por supuesto el cumplimiento de requerimientos adicionales. Así pues, para que la promesa de contrato sea obligatoria, además de constar por escrito, debe contener los requisitos para la validez de los actos y contratos. De tal manera que para perfeccionar el contrato solo falte la tradición de la cosa o las formalidades, esto según lo establecido en el artículo 1611 del Código Civil.

Dado lo anterior, el perfeccionamiento del contrato requiere cumplir con las condiciones de la promesa en general y con las formalidades que ha estipulado la ley para cada caso de contrato.

\section{ANÁLISIS DEL CONTRATO DE CONSUMO}

Las necesidades actuales del mercado enfrentan el derecho al reto de reconocer situaciones creadoras de obligaciones, como las relaciones de consumo, por las cuales entra en vigencia el contrato de consumo, que en su gran mayoría es celebrado mediante la utilización de proformas o contrato de adhesión.

18 Véase. M. González Pernett, supra, nota 8. 
Es importante observar que el contrato de consumo está conformado por dos partes: el productor o proveedor y el consumidor; este último es definido en el artículo 5 número 3 del Estatuto del Consumidor ${ }^{19}$ como:

Toda persona natural o jurídica que, como destinatario final, adquiera, disfrute o utilice un determinado producto, cualquiera que sea su naturaleza para la satisfacción de una necesidad propia, privada, familiar o doméstica y empresarial cuando no esté ligada intrínsecamente a su actividad económica. Se entenderá incluido en el concepto de consumidor el de usuario.

Frente a la definición de contrato de consumo, Rinnessi ${ }^{20}$ expresa que:

El contrato de consumo es el celebrado entre un consumidor final - persona física o jurídica_ con una persona física o jurídica, pública o privada, que actuando profesional u ocasionalmente, en calidad de productora, importadora o distribuidora, comercialice bienes o preste servicios, y que tenga por objeto la adquisición, uso o goce de estos por parte del primero, para su uso privado, personal o familiar.

Otro aspecto fundamental del contrato de consumo es bilateral y está basado en relaciones de consumo, siendo lo normal que el productor —-mediante contratos preestablecidos - realice la oferta ya sea en exhibiciones al público en general o por internet. En esta dirección, la definición de consumidor coincide con la postura adoptada por la Corte desde el año 2005, en la que se concibe al consumidor como el destinatario final del bien ${ }^{21}$.

En efecto, la Corte Constitucional, en la Sentencia C-1141 de 2000, planteó que el contrato con el consumidor "constituye una particular categoría que surge entre quienes se dedican profesionalmente a elaborar o proveer bienes o prestar servicios con quien los adquiere con el fin de consumirlos".

19 Ley 1480 de 2011 [Congreso de la República]. Por medio de la cual se expide el Estatuto del Consumidor y se dictan otras disposiciones. Octubre 12 de 2011.

20 Cf. Juan Antonio Rinnessi. RELACIÓN DE CONSUMO Y DERECHOS DEL CONSUMIDOR. Astrea. (2006).

21 Corte Constitucional de Colombia. SENTENCIA C-072/2005. (MP César Julio Valencia Copete; 03 de mayo de 2005). 
I gualmente, la Corte señala en la Sentencia T-145 de 2004 que: "Se consagró en el artículo 78 que los derechos de los consumidores han de ser considerados derechos colectivos"; y en la Sentencia C-1141 de 2000 señala que:

[...] es precisamente el consumidor quien, por encontrarse en condiciones de vulnerabilidad económica y de desequilibrio, es destinatario de una especial protección normativa; por supuesto que la profesionalidad del productor, que lo hace experto en las materias técnicas y científicas en torno de las cuales realiza su labor, su sólida capacidad económica, su vocación para contratar masivamente, las modalidades de contratación a las que acude, entre muchas otras peculiaridades, lo sitúan en un plano de innegable ventaja negocial que reclama la intervención de legisladores y jueces con miras a restablecer el equilibrio perdido.

De conformidad con lo anterior, manifiesta Herrera ${ }^{22}$ en su tesis doctoral que

[...] pueden identificarse tres (3) diferentes vías de perfeccionamiento: a) El consentimiento, como reflejo de una oferta y aceptación, propio de los contratos paritarios; b) La adhesión a condiciones generales de contratación o a contratos formulario, que es necesaria para contratos estandarizados o masivos; y c) Las conductas socialmente típicas, que en una valoración objetiva dan a entender que las partes manifestaron su voluntad de obligarse.

\section{Características de los contratos de consumo}

Los contratos de consumo presentan unas características especiales que lo definen y diferencian de los demás tipos de contratos, y que son ampliamente conocidas por el proveedor o vendedor, pero no sucede lo mismo con el consumidor, lo que lo pone de entrada en desventaja en la relación comercial, incluso desde antes de su surgimiento.

Igualmente, figuras como el retracto, la garantía legal, la posibilidad de declaratoria de abusividad de las estipulaciones contractuales, la interpretación a favor del consumidor, entre otras instituciones que son propias del derecho de consumo, responden a sus desafíos.

22 Cf. Fredy Andrei Herrera Osorio. ELEMENTOS PARA UNA TEORía DE LA FORMACIÓN DEL CONTRATO PRIVADO CONTEMPORÁNEO. Universidad Nacional. (2015). 


\section{Contrato asimétrico}

El contrato de consumo es un contrato asimétrico, porque es un contrato entre desiguales pues una de las partes (que es el productor o vendedor) posee toda la información relevante para la celebración del contrato, y tiene mayor poder económico y de negociaciones que el comprador.

Es por esta asimetría que se ha generado la necesidad de normarlo en procura de proteger a los consumidores. Al respecto, Márquez ${ }^{23}$ expresa que se debe proteger al consumidor en razón de la desigualdad entre las partes, dado que el consumidor no posee la información del negocio a celebrar y obtenerla tendría un costo bastante alto.

Morales Acosta ${ }^{24}$ expresa: "Sin embargo, contrario a lo que pareciese, el contrato de adhesión o bajo condiciones generales de contratación es un mecanismo necesario en el mercado". Es necesario tener en cuenta que las principales circunstancias que rodean la asimetría contractual en las relaciones de consumo es el desequilibrio informativo. No obstante, González Bullard ${ }^{25}$ considera que "la información es un bien porque tiene un valor económico determinado por las circunstancias y por la utilidad que representa para los contratantes".

De allí se tiene en cuenta lo expresado por Palacio Gonzáles"26: "Se observa que la información despliega su eficacia en dos ámbitos diferenciados: por un lado, es vista como la forma de prevención de los clásicos vicios del consentimiento en cuanto tiene por objeto evitar el error en que puede incurrir un contratante inadecuadamente informado". Esto significa que en los contratos de consumo hay siempre una parte: el consumidor que no tiene el poder de contar con toda la información que requiere para tomar su decisión de compra y esta improbabilidad lo hace vulnerable frente a los productores. Por ello, la Corte Suprema de Justicia ${ }^{27}$ consagró en dicha dirección lo siguiente:

Precisamente, al amparo de este principio superior puede afirmarse que la tutela efectiva de los intereses de los consumidores y usuarios,

23 Cf. Felipe Márquez Robledo. APUNTES DE DERECHO ECONÓMICO Y DE LA COMPETENCIA. Universidad Javeriana. (2005).

24 Cf. Alonso Morales Acosta. Asimetría INformativa. Asesorandina. (2008).

25 Cf. Alfredo González Bullard. LA ASIMETRía dE INFORMACIÓN EN LA CONTRATACIÓN A PROPÓSITO DEL DOLO OMISIVO. Palestra. (2000).

26 Cf. María Dolores Palacio Gonzáles. El deber de información en los contratos con consumidores. En: Santiago Espiau Espiau y Antoni Vaquer Aloy (coords.). BASES DE UN DERECHO CONTRACTUAL EUROPEO. Tirant lo Blanch. (2002).

27 Corte Suprema de Justicia. Sala de Casación Civil. SENTENCIA DEL 7 DE FEBrERo DE 2007 (MP César Julio Valencia Copete; 07 de febrero de 2007). Exp. 23162-31-03-0011999-00097-01. 
habida cuenta de la posición de inferioridad o debilidad que ordinariamente ocupan en el tráfico mercantil y la asimetría que caracteriza sus relaciones jurídico-económicas con los distribuidores o fabricantes, no puede verse restringida o limitada por el principio de la relatividad de los contratos, cuyo alcance, por cierto, tiende cada vez a ser morigerado por la doctrina jurisprudencial, puesto que, con independencia del vínculo jurídico inmediato que ellos pudieran tener con el sujeto que les enajenó o proveyó un determinado bien o servicio, las medidas tuitivas propias de su condición han de verse extendidas hasta la esfera del productor o fabricante, como quiera que éste es quien ha gestionado, controlado o dirigido el diseño y elaboración del producto, entre otros aspectos, así como ha determinado ponerlo en circulación o introducirlo en el mercado, adquiriendo, por contera, un compromiso en torno de la calidad e idoneidad del mismo, por lo que, desde luego, no puede resultar ajeno o indiferente a sus eventuales defectos o anomalías, ni a los peligros o riesgos que estos pudieran generar, como tampoco a las secuelas de orden patrimonial que llegaren a afectar a su destinatario final — consumidores o usuarios - o a terceros, con lo que queda claramente establecida una "responsabilidad especial".

\section{Contrato de adhesión}

El contrato de adhesión, de acuerdo con Vallespinos ${ }^{28}$, se ha definido como "aquel en el cual el contenido contractual ha sido determinado con prelación, por uno solo de los contratantes al que se deberá adherir el cocontratante que desea formalizar una relación jurídica obligatoria".

La contratación por adhesión es consecuencia de los cambios sociales, económicos y políticos, que han generado una forma de contratación propia de la sociedad contemporánea, de la sociedad de consumo, en la cual se masificó la venta de bienes y la prestación de servicios. Al respecto, Arrubla ${ }^{29}$ justifica la elaboración de los contratos preestablecidos en razón a la contratación en masa, pues estos protegen al proveedor y hacen más fácil la negociación.

28 Cf. Carlos Gustavo Vallespinos. EL CONTRATO POR ADHESIÓN A CONDICIONES GENERALES. Universidad de Buenos Aires. (1984).

29 Cf. Jaime Alberto Arrubla Paucar. contratos mercantILES (TOMO III). Ediciones Jurídicas Dike. (2008). 
Sin embargo, es necesario tener en cuenta que existe diferencia entre el adherente y el consumidor, pues de acuerdo con lo que establece Vega ${ }^{30}$, "pese a proteger finalmente al consumidor no fueron creadas pensando en el ciudadano consumidor, es decir que no son suficientes porque no integran todos los principios y fundamentos propios de un estadio más evolucionado, el que corresponde al proceso evolutivo del consumerismo".

Estos contratos por adhesión deben contener toda la información que requiere el consumidor, siendo un requisito fundamental de estos. Así lo expresa la Corte Constitucional sobre los contratos que se realizan entre entidades del sistema financiero y los consumidores de sus productos y servicios:

El acceso completo, veraz y oportuno a la información —que es una condición elemental, inherente a toda actividad de consumo-adquiere especial trascendencia en el marco del sistema financiero, en razón a los contratos de adhesión que suelen ofrecer las entidades vigiladas en el mercado, a la complejidad de los términos contractuales que se manejan y al estado de indefensión en que se encuentran los usuarios. Siendo así, la información es una de las herramientas clave para empoderar al ciudadano en su ejercicio contractual, tanto antes de la celebración de un contrato, como durante su ejecución y aún después de la terminación del mismo, con el fin de precaver que la libertad contractual se emplee abusivamente en detrimento de otros derechos fundamentales. Es por ello que cualquier restricción injustificada al acceso a la información debe entenderse como una práctica abusiva, propiciada por el poder dominante del que gozan las entidades aseguradoras y bancarias ${ }^{31}$.

Igualmente, la Corte ha señalado que estos contratos de adhesión deben fundamentarse en la buena fe y tener un carácter restrictivo, tal como lo plantea en la Sentencia T-152 de 2006:

Teniendo en cuenta el principio de la buena fe y su carácter restrictivo en los contratos de adhesión, la Corte Constitucional ha precisado que en los contratos de Medicina Prepagada y de Seguros Médicos, al momento de realizar la afiliación o de suscribir el contrato, debe señalarse de manera

30 Cf. Yuri Vega Mere. contratos de consumo. Editora Jurídica Grijley. (2001).

31 Corte Constitucional de Colombia. SEnTENCIA T-136/2013. (MP Jorge Iván Palacio Palacio; 13 de marzo de 2013). 
taxativa y expresa las exclusiones médicas — entiéndase enfermedades o afecciones - respecto de las cuales no se dará cubrimiento alguno. Si esto no ocurre, no es posible que la compañía de medicina prepagada - la aseguradora puedan relevarse de la obligación de autorizar y cubrir los tratamientos o servicios médicos que el beneficiario o asegurador requieran, invocando o valiéndose de cláusulas ambiguas o generales, como por ejemplo aquellas que dicen excluir todas las enfermedades congénitas o todas las preexistencias.

La Corte es, por lo tanto, muy enfática al expresar la necesidad de proteger al consumidor de productos financieros, catalogando como prácticas abusivas todas aquellas tendientes a restringir la información, o a utilizar cláusulas ambiguas o generales, para vulnerar los derechos del consumidor. Dicha Sala considera que esta posición surge de un poder dominante, que no puede ser aceptado, porque es generador de desequilibrio y abuso del consumidor, pero ¿qué sucede en los otros sectores del mercado?

\section{Es un contrato normado}

El contrato de consumo es un contrato normado con un nivel de protección especial, debido a que no existe igualdad alguna entre los contratantes. Sin embargo, la protección efectivamente brindada a través del Estatuto del Consumidor es insuficiente, tal como lo expresa la Sala de Casación Civil de la Corte Suprema de Justicia en la sentencia con expediente 25899-3193-992-1999-00629-01 del 30 de abril de 2009:

La relación de consumo constituye una particular categoría que surge entre quienes se dedican profesionalmente a elaborar o proveer bienes o prestar servicios con quien los adquiere con el fin de consumirlos; y es precisamente el consumidor, quien, por encontrarse en condiciones de vulnerabilidad económica y de desequilibrio, es destinatario de una especial protección normativa; por supuesto que la profesionalidad del productor, [...], lo sitúan en un plano de innegable ventaja negocial que reclama la intervención de legisladores y jueces con miras a restablecer el equilibrio perdido. Las más relevantes reivindicaciones de los consumidores apuntan a la necesidad de reformular las reglas de formación del contrato de modo que abarquen las nuevas realidades negociales, concretamente, la contratación masiva; hacia la apremiante necesidad 
de consagrar un periodo de reflexión seguido del derecho de arrepentimiento del consumidor; a vigorizar la tutela de éste en relación con los vicios del consentimiento frente a las dificultades propias de la contratación masiva; a destacar la importancia de la publicidad y, en general, de la información en los procesos contractuales; a regular lo concerniente con las cláusulas limitativas de responsabilidad; a robustecer el régimen de responsabilidad del fabricante y los proveedores, entre otras.

En el mismo sentido, se debe normar y reglamentar la información en los contratos de consumo, porque la información es un factor fundamental y determinador de la manifestación de voluntad del consumidor. Esto debido a que mientras un consumidor bien informado toma su decisión razonablemente, un consumidor mal informado se obliga sin tener plena consciencia de las condiciones, características y consecuencias jurídicas del contrato que celebra por acción o inacción.

En lo que respecta a los contratos de prestación de servicios públicos, se han normado y protegido especialmente, tal como lo manifiesta la Corte en Sentencia C-1162 de 2000: no se ocasionan en razón a la autonomía de la voluntad, sino en razón al interés del Estado en cumplir sus fines satisfaciendo a los ciudadanos ciertas necesidades básicas.

Debido a ello, los contratos de consumo han sido especialmente reglados en lo que respecta a la protección que debe brindarse al consumidor. Al convertirse esta información en deber secundario, es un deber principal de las partes, especialmente por parte del productor o del comerciante que son quienes detentan la información. El incumplimiento de este deber ocasiona, entre otros factores, que el consumidor desconozca que a veces es necesario manifestar la voluntad so pena, valga decir, en la práctica, de tener que asumir obligaciones que no tenía contempladas.

\section{CONCLUSIONES}

El perfeccionamiento del contrato requiere cumplir con las condiciones de la promesa en general y cumplir con las formalidades que ha estipulado la ley para cada caso de contrato. Los contratos de consumo son asimétricos, porque el poder económico y la información son detentados por los productores o comerciantes frente a los consumidores, y ello genera la necesidad de normarlos y de producir cambios legislativos que provean de especial protección a estos últimos.

La contratación por adhesión es consecuencia de los cambios sociales, económicos y políticos, que han generado una forma de contratación propia de la sociedad 
contemporánea, de la sociedad de consumo, en la cual se masificó la venta de bienes y la prestación de servicios

El contrato de consumo es un contrato normado con un nivel de protección especial, debido a que no existe igualdad alguna entre los contratantes, porque los oferentes pueden manipular la información. Pero de acuerdo con la Corte, esta protección es insuficiente, dada la dinámica de las formas de ofrecer y comprar bienes de consumo, que evoluciona hasta el punto de que la legislación al respecto suele estar atrasada para generar el desequilibrio entre productores y/o comercializadores y consumidores.

\section{REFERENCIAS}

Alfredo Barros Errazuriz. CURSO DE DERECHO civil (VOL. III). Nascimento. (1932).

Allan Farnsworth. Contratos (Tomo I). Aspen Law \& Business Publisher. (1998).

Alonso Morales Acosta. Asımetría Informativa. Asesorandina. (2008).

Carlos Gustavo Vallespinos. El CONTRATO POR ADHESIÓN A CONDICIONES GENERALES. Universidad de Buenos Aires. (1984).

Christian Larroumet. Teoría General del Contrato (Vol. I). Temis. (1999).

Corte Constitucional de Colombia. SentenciA C-072/2005. (MP César Julio Valencia Copete; 03 de mayo de 2005).

Corte Constitucional de Colombia. Sentencia T-136/2013. (MP Jorge Iván Palacio Palacio; 13 de marzo de 2013).

Corte Suprema de Justicia. Sala de Casación Civil. SenTENCIA DEL 7 DE FEBRERO DE 2007 (MP César Julio Valencia Copete; 07 de febrero de 2007). Exp. 23162-31-03-001-1999-00097-01.

Decreto 410 de 1971 [Presidencia de la República de Colombia]. Por el cual se expide el Código de Comercio. Junio 16 de 1971.

Felipe Márquez Robledo. ApUnTES DE DERECHO ECONÓMICO Y DE LA COMPETENCIA. Universidad Javeriana. (2005). 
Fredy Andrei Herrera Osorio. ELEMENTOS PARA UNA TEORÍA DE LA FORMACIÓN DEL CONTRATO PRIVADO CONTEMPORÁNEO. Universidad Nacional. (2015).

Alfredo González Bullard. LA ASIMETRÍA DE INFORMACIÓN EN LA CONTRATACIÓN A PROPÓSITO DEL DOLO omisivo. Palestra. (2000).

Jaime Alberto Arrubla Paucar. Contratos merCANTILES (Tomo III). Ediciones Jurídicas Dike. (2008).

José Puig Brutau. Fundamentos DE DERECHO CIVIL. Marcial Pons. (1978).

Juan Antonio Rinnessi. ReLACIÓn DE CONSUmo y DERECHOS DEL CONSUMIDOR. Astrea. (2006).

Ley 1480 de 2011 [Congreso de la República]. Por medio de la cual se expide el Estatuto del Consumidor y se dictan otras disposiciones. Octubre 12 de 2011.

Luis Diez Picazo. Teoría del Contrato (Vol. 1). Civitas. (1993).

Manuel Albaladejo García. DeRECHO CIVIL, EL NEGocio JURídico. Bosch. (1994).

Manuel De la Puente y Lavalle. Estudios Sobre El Contrato PRIVAdo. Lima: T.I. (1983).

Manuel González Pernett. Contratos y obligaciones: LA OFERTA o PROPUESTA A CONTRATAR. (2010). Disponible en: http://migonzalezp.blogspot.com/2009/08/la-oferta.html

María Dolores Palacio Gonzáles. El deber de información en los contratos con consumidores. En: Santiago Espiau Espiau y Antoni Vaquer Aloy (coords.). BASES DE UN DERECHO CONTRACTUAL EUROPEO. Tirant lo Blanch. (2002).

Renato Scognamiglio. Teoría general Del CONTRATO. Universidad Externado de Colombia. (1996).

Roberto Ruggiero. INSTITUCIONES DE DERECHO CIVIL (TOMO II). Civitas. (1993).

Walter Kaune Arteaga. CURSO DE DERECHO CIVIL DE CONTRATOS. Zegada. (1981).

Yuri Vega Mere. Contratos DE Consumo. Editora Jurídica Grijley. (2001).

Thomas Hobbes. LEVIATÁN O LA MATERIA, FORMA Y PODER DE UNA REPÚBLICA ECLESIÁSTICA Y CIVIL. Fondo de Cultura Económica (2001). 